\title{
LAS REPRESENTACIONES SOCIALES DE LA POLITICA Y LA DEMOCRACIA
}

\author{
MANUEl CÁRDENAS, LUIS PARRA, JUAN PICÓN, \\ HÉCTOR PINEDA Y RODRIGO ROJAS*
}

\begin{abstract}
RESUMEN
Se presenta un estudio sobre las representaciones sociales de la política y la democracia, elaboradas por una muestra de jóvenes de entre 18 y 29 años de edad. Para el análisis de dichas representaciones se ha utilizado un método de asociación libre de palabras a un conjunto de estímulos y un cuestionario de evaluación, el cual toma la forma de un diferencial semántico. Los resultados obtenidos a través de la asociación libre de palabras son analizados por medio de métodos multivariados (análisis de conglomerados, escalamiento multidimensional y análisis de correspondencias). Los resultados del cuestionario de imagen son resumidos en cuatro factores principales cuyas medias son comparadas según diversos criterios de clasificación. El principal efecto observado es el rechazo, por parte de los sujetos de la muestra, a participar a través de los canales institucionales y por medio de métodos convencionales de acción política. Su acción nos indica que estos jóvenes, lejos de restarle importancia a la vida política, poseen un significado novedoso de lo político y que puede ser contrapuesto a las definiciones más tradicionales de la misma. Por otra parte, es evidente su desencanto con la democracia realmente existente, pudiendo rastrearse en sus relaciones cotidianas formas alternativas de organización y que consideran más cercanas a su versión de la democracia.
\end{abstract}

PALABRAS ClAVE: RePRESENTACiÓn SOCIAL, Política, JuVENTUd

* Docentes Escuela de Psicología, Facultad de Humanidades, Universidad Católica del Norte, Antofagasta, Chile. E-Mail: jocarde@ucn.cl. 


\section{RESUMO}

\section{AS REPRESENTAÇOES SOCIAIS DA POLÍTICA E DA DEMOCRACIA}

Este artigo apresenta um estudo das representações sociais da política e da democracia, elaboradas por uma amostra de jovens entre 18 e 29 anos de idade. Para a análise de tais representações utilizou-se um método de associação livre de palavras a um conjunto de estímulos e um questionário de avaliação, o qual toma a forma de um diferencial semântico. Os resultados obtidos através da associação livre de palavras são analisados por meio de métodos multivariados (análise de conglomerados, escalamento multidimensional e análise de correspondências). Os resultados do questionário de imagem são resumidos em quatro fatores principais, cujas médias são comparadas segundo diversos critérios de classificação. O principal efeito observado é a rejeição, por parte dos sujeitos da amostra, em participar através dos canais institucionais e por meio de métodos convencionais de ação política. Sua ação nos indica que estes jovens, longe de não conferir importância à vida política, concebem um significado novo do político e que pode ser contraposto às definições mais tradicionais. Por outro lado, é evidente seu desencanto com a democracia realmente existente, podendo identificar-se em suas relações cotidianas formas alternativas de organização, que consideram mais próximas de sua versão de democracia.

PALAVRAS-CHAVE: REPRESENTAÇÃo SOCIAL, POLÍTICA, JUVENTUDE

\section{Abstract \\ POLITICS SOCIAL REPRESENTATIONS AND DEMOCRACY}

This is a study of the social representations appears on the politics and democracy, elaborated by youth sample between 18 and 29 years old. For the analysis of these representations it has been used a method of free association of words as stimuli and one evaluation survey, that takes the form similar a semantic differential. The outputs obteined through the free association of words were analysed according of multivariated methods (hierarchical cluster analysis, multidimensional scaling and correspondences analysis). The results on the image survey were summarized in four principal factors and compares averages for multiple categorization. The principal observed effect is the rejection, on the part of the subjects of the sample, to taking part across the institutional channels and by means of conventional methods of political action. His action indicates us that these young men, far from reducing importance to the political life, possess a novel meaning of the political thing and that it can be opposed to the most traditional definitions of the same one. On the other hand, his disenchantment is evident with the really existing democracy, there being able to be traced in his daily relations alternative forms of organization and that they consider to be nearer to his version of the democracy.

Key WORdS: Social REPRESENTATION, Political, Youth 


\section{INTRODUCCIÓN}

LAS REPRESENTACIONES SOCIALES REFIEREN a formas de conocimiento elaboradas y compartidas al interior de un grupo que participa de prácticas sociales comunes y que tiene una determinada inserción en la estructura social (Moscovici, 1984; Jodelet, 1986). Estas representaciones orientarían la acción de los miembros de un determinado colectivo, prescribiendo comportamientos y condicionando adhesiones, toda vez que permitirían soportar la identidad de los mismos. De este modo, el contenido de las representaciones que los individuos poseen depende de los grupos y las relaciones sociales en que estos participan (Doise, 1991), no existiendo una representación homogénea para todas las personas, sino derivándose representaciones diferenciadas para los diversos contextos sociales, económicos y culturales existentes. Estas representaciones constituirían el campo psicológico interno (Galam y Moscovici, 1991) que delimita los grados de libertad que tendrán los sujetos a la hora de tomar decisiones y actuar.

Por otra parte, debemos tener claro que el calificativo social de una representación se deriva más bien de las funciones prácticas de la representación que de su origen compartido. Entre estas funciones se encuentra la de familiarizar a los sujetos con lo extraño y lo novedoso, permitiendo incorporar los esquemas extraños en aquellos preexistentes. Se trataría de un saber práctico, del saber del sentido común, que permitiría racionalizar las prácticas sociales toda vez que anclar lo nuevo en las categorías de pensamiento preexistentes, de modo de permitir al sujeto dominio sobre su ambiente y la anticipación de las conductas de los otros grupos. Es decir, produce y orienta las conductas y la comunicación social (Moscovici, 1979, 1984; Carugati \& Palmonari, 1991) de los miembros de un grupo, permitiéndonos asistir a la construcción social de la realidad.

En relación a los conceptos que nos ocupan (los discursos sobre la política y la democracia) podemos considerar que existen diversos significados asociados a estos, de modo tal que más allá de las definiciones académicas disponibles podemos encontrar versiones simplificadas de dichos conceptos que designan el modo en que el sentido común las utiliza y entiende (y que en muchas ocasiones tienen un contenido y una lógica propia, más idiosincrásica, aunque no por ello menos ideológica), y que condicionan fuertemente el sentido de sus actuaciones. Este segundo nivel discursivo al que aludimos con el concepto de representación social se basa en la circulación de la in- 
formación disponible al interior de los grupos y en las comunicaciones informales de sus miembros, presentando por ello un carácter dinámico y diverso, y permitiendo justificar ciertos rumbos de acción (Echebarría, Fernández y González, 1993; Echebarría y Álvarez, 1996) y coordinar las acciones de los individuos con las de su grupo mediante un proceso de reconstrucción de los objetos sociales (Jodelet, 1986). Es justamente aquí donde se torna relevante considerar la diferente implantación social de los sujetos, sus diversas características demográficas y sus diferentes orientaciones ideológicas.

Para el estudio que ahora presentamos hemos considerado relevante analizar las representaciones que los jóvenes elaboran de la política y la democracia, en tanto consideramos que de su comprensión se podrían obtener datos respecto de la baja relevancia asignada por buena parte de este segmento social a una forma particular de entender estos conceptos, así como de las pautas de acción que se derivan de dicha forma de entender la relación entre participación política y democracia.

Parece ser un hecho que en nuestro país la relación existente entre los jóvenes y la política puede caracterizarse por un profundo quiebre y distanciamiento de éstos respecto de las actividades y canales convencionales de participación, así como de las formas institucionales de acción. Lo anterior ha sido frecuentemente interpretado como un proceso de desencanto masivo, cuando no como anomia o simple desidia. Lejos de esta posición, tenemos la firme convicción que si atendemos al discurso juvenil podremos observar la existencia de prácticas sociales enraizadas firmemente en una concepción de la democracia radicalmente diferente a la ofrecida por los ideólogos del sistema (y que indicaría el agotamiento de la legitimidad de la democracia representativa en buena parte de este segmento social) y de prácticas políticas (entendidas como tal por resultar de un ejercicio comunitario realizado en el plano público) divergentes, no convencionales y menos institucionalizadas. De la polisemia de los términos que nos interesan no sólo se derivan diversos cursos de acción, sino que se pueden deducir diversos modos de relación comunitaria, así como una amplia gama de prácticas sociales dentro de las cuales los sujetos se encuentran inmersos.

Es por lo anterior que creemos resueltamente que para comprender la relación entre los jóvenes con la política debemos situarnos en el contexto histórico y social que les ha tocado habitar y que se encuentran directamente vinculados con la transformación de la estructu- 
ra institucional y de los cambios en el modelo económico y político chileno, del que han resultado importantes transformaciones sociales y culturales que han modificado las formas de interacción de la sociedad con la política, así como también las representaciones que ésta ha ido construyendo en torno a ella. Estos cambios en los términos de la relación pueden resumirse en grandes rasgos como pérdida de confianza en el rol del estado como canal privilegiado de la transformación social y por el cuestionamiento de la alternativa institucional como espacio predilecto para la participación social. En fin, se trata de la constatación de fracaso de la lógica de la asimilación que es vivida como un atentado a la identidad del actor juvenil en tanto niega el reconocimiento de la diversidad del mismo (Sandoval, 2003).

Este alejamiento de lo institucional y la invención de nuevos canales de participación del actor juvenil han contribuido a restar legitimidad a ciertas prácticas tradicionales de expresión política (tales como la conducta de voto y la afiliación a partidos políticos) y a perfilar nuevas formas de asociatividad que, aunque no contengan un discurso político explícito, apuntan a construir formas de asociación y autogestión de la vida comunal de los actores juveniles. Es decir, prácticas históricamente consideradas privadas e incluso lesivas de «la vida política» (que van desde la participación en grupos de interés hasta la desordenada irrupción en el espacio público con demandas que la mayor parte de las veces no hacen sino expresar la frustración y el desencanto de los jóvenes con un modo particular de entender lo político) pueden expresar un proyecto propio que puede ir desde la participación no institucional y no convencional, hasta una suerte de «nomadismo» que los ha hecho instalarse en los márgenes del sistema político en vistas a la construcción de espacios «más democráticos» y de una nueva acumulación de legitimidad para su proyecto alternativo.

Creemos estar en presencia de un actor que construiría su identidad a partir de su participación en formas de asociación o agrupamiento menos formales, poco jerárquicos y más participativos. Estos modos de agenciamiento juvenil generarían unas representaciones sobre la política en estrecha relación con su proyecto participativo y autogestionario. Como verá el lector, nada más alejado de la versión que muchos actores institucionales fomentan, y que se encuentran basadas en un prototipo juvenil despolitizado e inconsistente, disruptivo e irracional. Es decir, se trataría de la opción por el éxodo a la vida comunitaria y a la contracultura urbana, así como por la construcción de modos de relación que no dejan de ser políticos (en tanto preocupa- 
ción y participación en la gestión de los asuntos de la comunidad) aunque sí pretendidamente democrático (por la vía de la participación simétrica al interior de la comunidad y de la eliminación de los excesivos controles normativos). Este éxodo puede constatarse entre otras cosas en la continua disminución en el padrón electoral (INJUV, 2003) y en cambios en los modos de organización, formas de participación y tácticas de acción.

Ahora bien, lejos de caer en una idealización del actor juvenil y en la homogeneización de un actor por definición diverso y heterogéneo (Dávila, 2004), pretendemos dar cuenta de las diversas formas de respuesta a una institucionalidad que resulta abrumadora y que es vivenciada como excluyente e inútil, cuando no derechamente represiva. De este modo, es probable que nos encontremos con representaciones diversas que podrán ir desde la creencia en que la obtención de derechos sociales y la movilidad social depende más de los propios recursos y capacidades que de su participación activa en la política tradicional (INJUV, 2003), hasta aquellas ya descritas y que tienen un proyecto político - explícito o no- basado en la construcción de comunidad y en la multiplicación de espacios democráticos, pasando por aquellas en que la política sólo es una forma más dentro de la oferta existente desde donde pensar la propia identidad (Garretón y Villanueva, 1999). Tomadas en conjunto, esta diversidad de representaciones expresarían un cuestionamiento por parte de los jóvenes de la efectividad del espacio institucional para satisfacer sus necesidades y demandas. Para efectos de este estudio intentaremos indagar en los discursos asociados a formas de participación política no convencional y extrainstitucional, además de describir y comparar las representaciones sociales existentes que poseen diferentes grupos de jóvenes sobre la política y la democracia, asumiendo que la diferente implantación social de los sujetos determinará representaciones sociales diferenciadas, de las que se derivarán comportamientos específicos que serán prescritos por dichas representaciones y que constituirán formas diversas de acercarnos al fenómeno de lo político.

\section{MÉTODO}

a) Muestra

La muestra quedó conformada por 165 jóvenes, 69 hombres $(41.8 \%)$ y 96 mujeres (58.2\%) cuyas edades fluctuaban entre los 18 y los 29 años 
(la media de edad fue de 21.79 años y la desviación típica de 2.83 ). La mayor parte de los participantes en el estudio eran solteros $(94.5 \%)$ y su actividad más frecuente era la de estudiantes universitarios (61.2\%). El 32.1\% se encontraba inscrito en los registros electorales, aunque sólo el $29.7 \%$ de estos votó en la última elección presidencial. Todos los participantes vivían en la ciudad de Antofagasta.

\section{b) Instrumento}

El instrumento quedó constituido por tres bloques. El primero de ellos contenía diversas preguntas que apuntaban a obtener información sociodemográfica de los participantes del estudio (sexo, edad, nivel socioeconómico, preferencias políticas, etc.) y que incluía, además, preguntas conducentes a indagar en su participación o afiliación en diversos grupos o asociaciones (partido político, grupo cultural, club deportivo, etc.), así como una escala en la que se les consultaba por el grado de confianza que tenían en diversas instituciones (gobierno, sindicatos, juntas de vecinos, etc.) y cuyos valores oscilaban entre 1 (nada de confianza) y cuatro (mucha confianza).

La segunda parte del instrumento quedó constituida por una escala de 16 ítems construida para el concepto de política y que toma la forma de un diferencial semántico (Osgood, Suci y Tanembaum, 1976). Los ítems de la escala final se seleccionaron a partir de los análisis realizados sobre una muestra piloto por medio de un análisis factorial (método de componentes principales con rotación VARIMAX) y que determinó la presencia de cuatro factores principales: estilo de ejercicio (25.08\% de la varianza), estructura (11.49\% de la varianza), evaluación de la eficacia (10.45\% de la varianza) e integración $(7.68 \%$ de la varianza). Cada una de las dimensiones quedó compuesta por cuatro ítems. En conjunto estos cuatro factores explicaban el 54.76\% de la varianza total (índices de adecuación de la muestra: KMO de 0.84 y prueba de esfericidad de Bartlett con significación $=0.000$ ). El diferencial semántico utilizado toma puntajes que van entre -3 y 3 .

Finalmente, la tercera parte del instrumento quedó conformada por un protocolo verbal en que se ofrecían a los sujetos diez conceptos para que asociaran libremente palabras a ellos. Los conceptos utilizados son: poder, comunidad, partidos políticos, elecciones, democracia, política, movimientos sociales, protesta, orden y juventud. Este método trabaja sobre la base del supuesto que las personas tienen un número limitado de significados asociados a un determinado objeto de re- 
presentación, que los contenidos de dicha representación están estructurados jerárquicamente sobre la base de su semejanza o distancia con otros significados, y que la estructura de dicha representación está orientada por pocas dimensiones de actitud (Doise, Clémence y Lorenzi-Cioldi, 1993; Cárdenas y Blanco, 2004, 2006).

En su conjunto, los análisis realizados sobre los diccionarios generados por el procedimiento asociación libre nos permitirán observar cómo se posiciona nuestra muestra en referencia a dichos términos. Los diccionarios han sido creados siguiendo ciertas reglas de procedimiento: a) las palabras son reducidas a su forma singular; b) las diferentes formas de un palabra son reducidas a la forma más común presente en el diccionario; c) los adverbios y sustantivos son reducidos a su correspondiente adjetivo; d) las combinaciones de palabras son reducidas al correspondiente adjetivo, en caso de que este exista en el diccionario (en caso contrario son mantenidas); e) las jergas son reducidas al adjetivo más cercano posible; f) las palabras que tienen obviamente un mismo sentido son reducidas a la forma más común en la muestra (Di Giacomo, 1980, 1987; Cárdenas, 2006).

\section{Resultados}

Comenzaremos con la descripción de los resultados obtenidos mediante el cuestionario sociodemográfico y por medio de la escala de confianza, de forma de poder entregar alguna información relevante sobre las diferencias existentes en nuestra muestra y sobre sus posiciones y afiliaciones. En términos generales, se puede afirmar que se trata de una muestra bastante diversa en términos de gustos y grado de asociatividad. Así, podemos constatar que el $45.5 \%$ de los jóvenes encuestados reporta no participar de ningún grupo o asociación. El porcentaje restante participa prioritariamente de «grupos culturales» $(13.3 \%)$, de algún «club deportivo» (10.3\%), «voluntariado» en alguna asociación u ONG $(9.1 \%)$, «grupo religioso» $(9.1 \%)$, «centro de alumnos» $(7.9 \%)$, «colectivo político» $(7.3 \%)$ o simplemente en algún «grupo de esquina» $(6.1 \%)$. Sólo un pequeño porcentaje se encuentra afiliado a algún «partido político» $(2.4 \%)$ o reporta participar en alguna «junta de vecinos» $(1.8 \%)$. Ninguno de entre los que trabajaba se encontraba sindicalizado.

Cuando se les pide posicionar políticamente sus ideas dentro de un continuo (el que originalmente tenía seis puntos, y que iba desde la «extrema derecha» a la «extrema izquierda», pero que ha sido reduci- 
do a tres niveles), el $45 \%$ de los sujetos se identifican con la izquierda, el $35 \%$ lo hace con posiciones de centro y el $20 \%$ describe sus ideas como de derecha.

En la Tabla 1 podemos apreciar los porcentajes obtenidos por medio de nuestra «escala de confianza en la instituciones». Resulta llamativo constatar el bajo nivel de confianza obtenido por casi todas ellas, en particular por el «gobierno», donde el 54.8\% reporta tener poca o nada de confianza en éste. Lo mismo ocurre con los «partidos políticos» $(86.1 \%)$, los «tribunales» $(71.1 \%)$, la «iglesia» $(66.8 \%)$, la «municipalidad» $(65.5 \%)$, las «juntas de vecinos» $(60.8 \%)$. Por otra parte, las instituciones mejor evaluadas son los «sindicatos», donde el $68.3 \%$ manifiesta relativa o mucha confianza (aunque nadie está sindicalizado, o quizás precisamente por ello), las «organizaciones sociales de base» $(60.9 \%)$, «carabineros» $(60.6 \%)$ y los «centros de alumnos» $(60.1 \%)$. Cabe destacar también la relativa confianza que la muestra manifiesta hacia los «medios de comunicación (45.5\%). Aunque, como veremos a continuación, todos estos resultados varían levemente al segmentar la muestra, ya sea por sexo, nivel socioeconómico (NSE) o posición política de los participantes.

Tabla 1

Escala de confianza

(en porcentajes)

\begin{tabular}{|l|c|c|c|c|}
\hline Instituciones & Nada & Poca & Relativa & Mucha \\
\hline Gobierno & 15.2 & 39.6 & 40.9 & 4.3 \\
\hline Centros de alumnos & 8 & 32.7 & 53.1 & 6.2 \\
\hline Sindicatos & 5 & 26.7 & 59.6 & 8.7 \\
\hline Iglesia & 38 & 28.8 & 27 & 6.1 \\
\hline Juntas de vecinos & 18.6 & 42.2 & 32.9 & 6.2 \\
\hline Carabineros & 10.3 & 29.1 & 49.1 & 11.5 \\
\hline Municipalidad & 20 & 45.5 & 33.9 & 0.6 \\
\hline Tribunales & 26.8 & 43.3 & 29.3 & 0.6 \\
\hline Congreso & 33.3 & 43.6 & 23 & 0 \\
\hline Partidos políticos & 38.8 & 47.3 & 13.9 & 0 \\
\hline Medios comunicación & 15.8 & 35.8 & 45.5 & 3 \\
\hline Organizaciones sociales de base & 9.3 & 29.8 & 49.1 & 11.8 \\
\hline
\end{tabular}


Al segmentar los grupos según la posición política reportada por los sujetos encontramos diferencias significativas en cuatro instituciones, a saber: iglesia $\left[\mathrm{F}_{(2,158)}=5,235 ; \mathrm{p}<0.05\right]$, carabineros $\left[\mathrm{F}_{(2,156)}=4,124 ; \mathrm{p}\right.$ $<0.05]$, medios de comunicación $\left[\mathrm{F}_{(2,159)}=4,869 ; \mathrm{p}<0.01\right]$ y organizaciones sociales de base $\left[\mathrm{F}_{(2,155)}=4,273 ; \mathrm{p}<0.05\right]$. Estas diferencias, puestas de relieves por las comparaciones de medias, indican que los sujetos de izquierda evalúan significativamente más mal a dichas instituciones, o mejor dicho, manifiestan niveles de confianza significativamente más bajos (salvo para el caso de las organizaciones de base, donde su media es más elevada). Es decir, la gente de derecha cree y confía más que la de izquierda en la iglesia, carabineros y en los medios de comunicación. Contra ello, los de izquierda parecen confiar más en las instancias que perciben como más cercanas y donde el control de la gestión y la participación parecen ser más directos. La gente que toma posturas de centro tiene a obtener puntuaciones intermedias que no la diferencian significativamente de ninguno de los dos grupos analizados.

Al segmentar la muestra por NSE nos encontramos algunas diferencias dignas de ser destacadas. Básicamente podemos informar de los niveles de confianza más elevados por parte de los sujetos de NSE bajo tanto para el gobierno $\left[\mathrm{F}_{(2,163)}=8,713 ; \mathrm{p}<0.001\right]$, el municipio $\left[\mathrm{F}_{(2,164)}=4,914 ; \mathrm{p}<0.01\right]$ y los medios de comunicación $\left[\mathrm{F}_{(2,164)}=3,513\right.$; $\mathrm{p}<0.05$ ] (en comparación con los sujetos de NSE medio y alto). Los participantes de NSE alto manifiestan significativamente más confianza en el Congreso $\left[\mathrm{F}_{(2,164)}=3,477 ; \mathrm{p}<0.05\right]$ que los grupos de NSE medio y bajo. Estas diferencias podrían explicarse por el propio modelo nacional basado en el Estado de Bienestar, en donde las instituciones del Estado se relacionan con los más pobres de forma asistencialista, y donde los restantes "poderes del Estado» son percibidos como más lejanos de los intereses de los ciudadanos.

Al realizar una segmentación de la muestra por sexo, nos encontramos que las mujeres confían significativamente más que los hombres en casi todas las instituciones evaluadas, salvo en el caso de los centros de alumnos, sindicatos, juntas de vecinos y organizaciones sociales de base, en las cuales no se encuentran diferencias estadísticamente significativas.

Pasamos ahora a revisar los resultados obtenidos por medio del diferencial semántico. Las medias del puntaje total para la escala del conjunto de los sujetos nos entregan información en sentido de mostrarnos que la evaluación general de la política es en términos gruesos 
negativa (media $=-0.51$ y desviación típica $=0.89$ ). Si bien debemos ser cautos a la hora de analizar este resultado, podemos desde ya observar la tendencia de los sujetos a asociar la actividad política con los significados negativos que se les han propuesto sobre esta. En la Tabla 2 encontramos los estadísticos descriptivos (media y desviación típica) para los 16 ítems. Las puntuaciones positivas remiten al primer adjetivo de cada par, y a mayor puntuación se supondría más fuerza en la dirección señalada por el signo (hemos ordenado los pares de forma de presentar los adjetivos que evalúan positivamente la política al lado izquierdo, por lo que signos positivos apuntarán a evaluar positivamente el concepto ofrecido).

Tabla 2

Estadísticos descriptivos (diferencial semántico)

\begin{tabular}{|l|c|c|}
\hline Política & Media & Desviación Típica \\
\hline Eficaz - Ineficaz & -0.28 & 1.81 \\
\hline Dinámica - Estática & -1.13 & 1.64 \\
\hline Comunitaria - Individualista & -0.76 & 1.76 \\
\hline Flexible - Rígida & -1.22 & 1.56 \\
\hline Efectiva - No efectiva & -0.76 & 1.79 \\
\hline Importante - Intrascendente & 1.53 & 1.70 \\
\hline Tolerante - Intolerante & -0.49 & 1.63 \\
\hline Necesaria - Innecesaria & 1.40 & 1.86 \\
\hline Descentralizada - Centralizada & -1.33 & 1.76 \\
\hline Democrática - Autoritaria & -0.01 & 1.75 \\
\hline Pluralista - Totalitaria & -0.34 & 1.61 \\
\hline Honesta - Corrupta & -1.47 & 1.58 \\
\hline Cercana - Lejana & -1.18 & 1.62 \\
\hline Accesible - Inaccesible & -0.48 & 1.75 \\
\hline Participativa - Excluyente & -0.66 & 1.73 \\
\hline Integradora - Discriminadora & -1.00 & 1.52 \\
\hline
\end{tabular}

Lo primero que llama la atención al observar los resultados es la mala evaluación general que la muestra con la que trabajamos realiza de la política, atribuyéndosele rasgos tales como rigidez, falta de dinamis- 
mo, excesivo centralismo, altos niveles de corrupción, lejanía y un marcado carácter discriminador. Eso sí, se le ve como una actividad importante y necesaria (que son los dos únicos puntajes positivos). Ahora bien, es importante señalar que la desviación típica es, para todos los ítems, bastante alta. Es cierto que una «variabilidad alta» es un hecho relativo, pero al menos debemos atender al hecho que existe una alta dispersión de las respuestas de los sujetos. Esta heterogeneidad de juicios implica que sería pertinente realizar una segmentación de la muestra y comparar si existen diferencias significativas entre los grupos que hemos venido utilizando (posición política, NSE y sexo), ya que la variabilidad de los rangos de respuesta puede indicar la presencia de categorías cruzadas (ser jóvenes es una categoría importante en términos de identidad, pero se revela la presencia de otras que funcionan de manera solapada a esta). Antes de realizar dicha segmentación ofrecemos la gráfica para las cuatro dimensiones detectadas por el instrumento (Figura 1).

Como se puede apreciar el factor eficacia es el mejor evaluado $(\mathrm{M}=0.47)$, lo que de todos modos sigue siendo muy bajo. En este sentido la muestra nos manifiesta el bajo grado de eficacia y efectividad que percibe en la política donde se resalta la importancia y la necesidad de dicha actividad. Es decir, existe la percepción de que la política sería relevante y necesaria, toda vez que poco eficaz. De allí resulta tal vez su negativa a participar en ella por las vías convencionales, ya que si la percepción de la eficacia resulta baja los jóvenes no consentirían en participar en una actividad que intuyen no les conducirá a lograr sus objetivos. Los restantes factores logran puntaciones negativas, lo que indicaría que tanto el estilo de ejercicio $(\mathrm{M}=-0.78)$, la estructura $(\mathrm{M}=-1.03)$ y los potenciales de integración $(\mathrm{M}=-0.69)$ de esta actividad son puestos en cuestión. De lo anterior se deduce una imagen sumamente devaluada de la actividad política, la que sería considerada como burocrática y deshonesta, lejana e individualista, excluyente y poco accesible, rígida y en exceso centralizada. Como vemos, el conjunto de la muestra significa de forma muy negativa la actividad política. Veamos ahora qué ocurre cuando segmentamos la muestra por medio de ciertos criterios de clasificación.

Al realizar comparaciones teniendo como criterio de segmentación el sexo no encontramos diferencias estadísticamente significativas ni para el puntaje total de la escala, ni para ninguna de las cuatro dimensiones. Tampoco aparecen diferencias en los ítems, por lo que podemos concluir que los significados asociados a la política son ne- 
gativos tanto para hombres como para mujeres, no siendo el sexo una categoría pertinente a la hora de evaluar este concepto. Lo mismo ocurre al ocupar como criterio el NSE de los sujetos, no se observan diferencias significativas ni en el puntaje total de la escala, ni en alguna de las dimensiones, ni en ninguno de los dieciséis ítems. Este último aspecto es relevante, pues cuestiona en buena medida la idea rectora de este estudio y que apuntaba a la aparición de diversas representaciones sociales según fuese la particular implantación social de cada grupo.

Figura 1

Perfil de puntaciones para cada factor

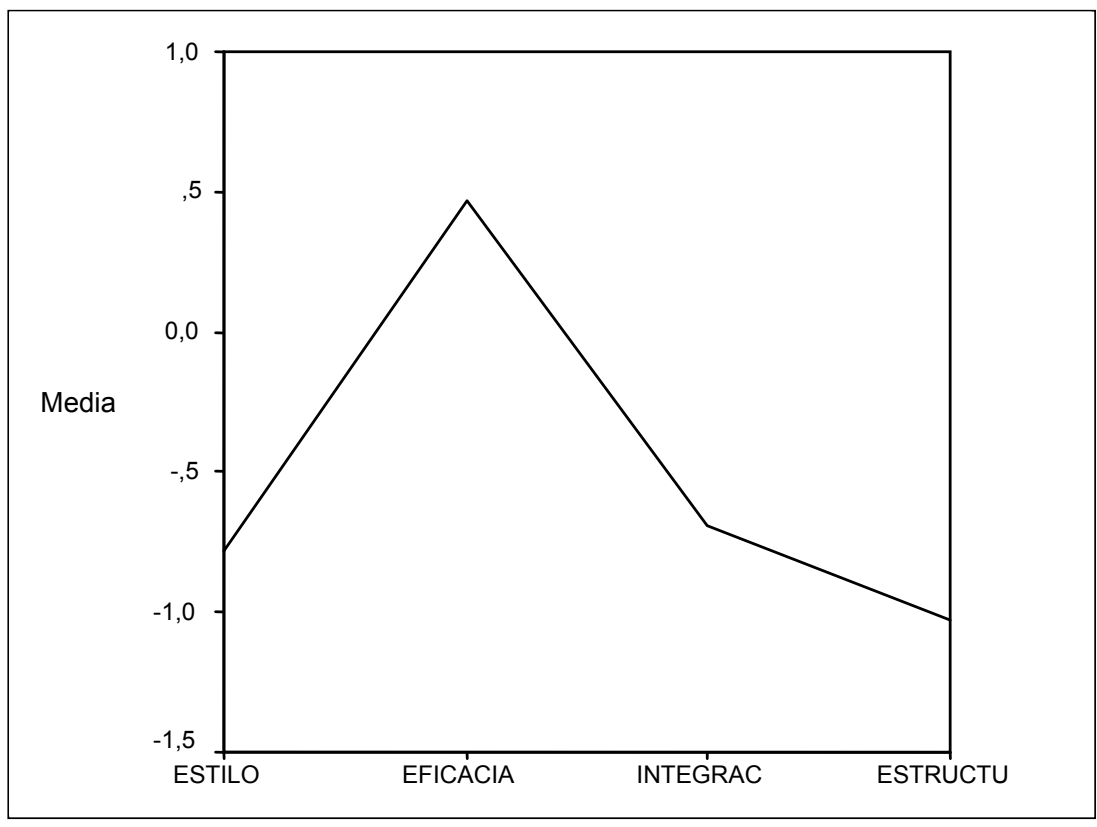

Al comparar a los sujetos inscritos en los registros electorales con los no inscritos sólo hallamos diferencias a nivel de uno de los ítems. Aquellos que no se encuentran inscritos evalúan la política como significativamente más corrupta que aquellos que están inscritos $\left[\mathrm{T}_{(76,196)}=3,354 ; \mathrm{p}=0.001\right]$. Lo mismo ocurre cuando el criterio de segmentación es si han votado o no en la última elección, es decir, aquellos que no votan consideran la política como menos honesta $\left[\mathrm{T}_{(156)}=-\right.$ $2,106 ; \mathrm{p}<0.05]$, toda vez que más flexible $\left[\mathrm{T}_{(67,001)}=3,661 ; \mathrm{p}=0.000\right]$. 
Este último dato resulta extraño y podría significar que para aquellos que no votan, y quizás justamente por ello, la actividad política parece poco rígida. También podría indicar que justamente la falta de oferta de discursos diferenciados y taxativos es la que impulsa a los jóvenes a restar su participación, en sentido de que podrían sentir que en general los grandes cambios y transformaciones no se dirimen en la actividad política institucional. Es decir, la diferencia entre las diversas ofertas políticas sería de matices y justamente sería esto lo que podría estar atentando contra la participación juvenil, ya que existiría el sentimiento de que la conducta de voto no sería capaz de propiciar cambios importantes ni en la estructura de repartición del poder ni en la forma de gestión del modelo económico y político.

Finalmente, al segmentar la muestra por posición política nos encontramos nuevamente con sorpresas. Los sujetos de todas las tendencias ideológicas evalúan negativamente la política, encontrándose diferencias sólo en un ítem, que daría cuenta del grado de centralización - descentralización percibido por los participantes. En concreto nos referimos a que los sujetos de izquierda consideran mucho más centralizada la política $\left[\mathrm{F}_{(2,157)}=4,123 ; \mathrm{p}=0.05\right]$ que aquellos de centro y derecha, lo que nos daría al mismo la pauta de su propia posición respecto de la estructura percibida de la política y de su reclamo de mayor descentralización y participación. En conjunto, tanto este dato como el de la inexistencia de diferencias por NSE cuestionarían la idea de representaciones diferenciadas y nos hablarían de una vivencia trasversal a las diversas categorías de jóvenes.

Por lo que respecta a los datos obtenidos por medio del procedimiento de asociación libre de palabras el primer paso ha consistido en la creación de un diccionario de asociación para los diez conceptos ofrecidos a la muestra. La producción total de palabra ascendió a la cifra de 1312 términos, lo que equivale a 21,87 términos por persona $(\mathrm{N}=60)$ y a un promedio de 2,18 términos por estímulo para cada sujeto. De este total de palabras hemos reducido la cifra a 418 términos (siguiendo los criterios comentados en el apartado que describe el instrumento), que son los que se utilizarán como diccionario definitivo. Posteriormente, sobre una matriz binaria (presencia o ausencias significadas por medio de 1 y 0 respectivamente) y sobre la base de una matriz de distancias generadas a partir de los datos, hemos realizado un análisis de conglomerados jerárquicos y un escalamiento multidimensional. 
Para el caso del análisis de conglomerados, dicha matriz se ha realizado sobre medidas de similitud utilizando un coeficiente Phi (cuatro puntos), que no es otra cosa que una versión binaria del coeficiente de correlación de Pearson. El método de aglomeración utilizado es el de vinculación intergrupos o promedio, el que permite aprovechar la información de todos los miembros del conglomerado que se compara (Pardo y Ruiz, 2003), calculándose la distancia promedio existente entre todos los pares de elementos de cada conglomerado. En la Figura 2 podemos observar el dendograma en que quedan representadas las etapas del proceso de fusión y las distancias existentes entre los elementos fundidos en cada etapa (reescaladas en una medida estandarizada de 25 puntos).

Figura 2

Análisis de conglomerados jerárquicos

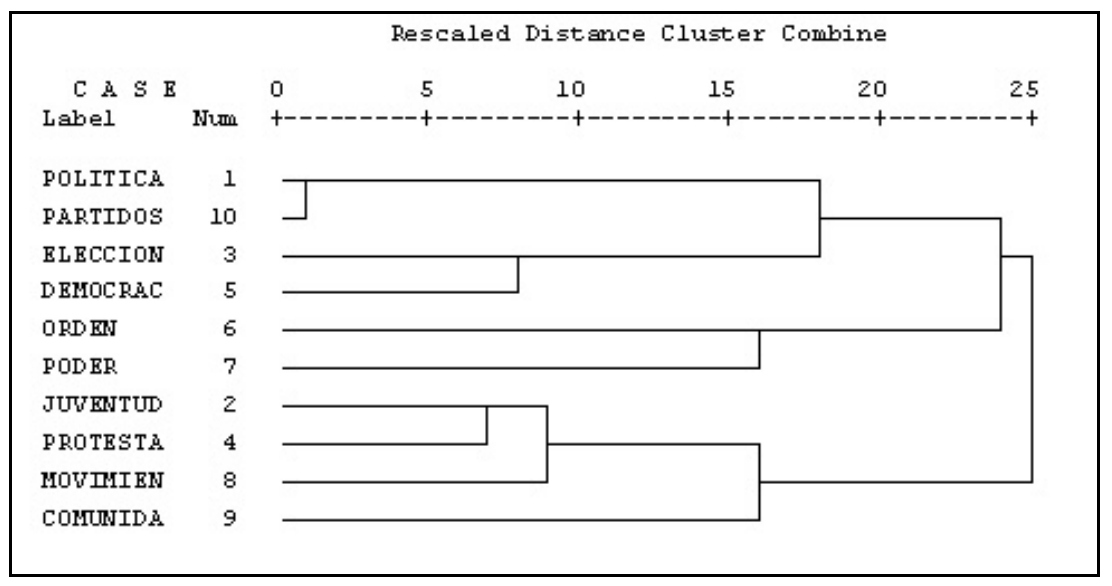

Como podemos apreciar, se observa cómo la muestra asigna una alta homogeneidad a los conceptos de política y partidos políticos. También se observa una fuerte asociación semántica entre elecciones y democracia, así como entre juventud y protesta. En términos generales podemos apreciar dos campos semánticos claramente diferenciados, aquel que vincula términos como política, partidos, elecciones, democracia, orden y poder (aunque estos dos últimos aglomeran bastante tarde con este polo), y otro - contrapuesto a éste- que vincula semánticamente a los jóvenes con la protesta, los movimientos sociales y la idea de comunidad. El cuadro general resulta bastante interesante, 
ya que de un lado los jóvenes ubican todos aquellos conceptos vinculados a la actividad política convencional, significando conceptos como democracia y política en su sentido más institucionalizado. La democracia como un ritual del voto y la política como una actividad profesional vinculada a los partidos políticos. Decíamos que resulta interesante el hecho de que el poder y el orden conglomeran con este polo de forma tardía, pudiendo esto significar que los verdaderos actores en este juego son agentes que imponen reglas de orden y manejan el poder desde un lugar no tan institucionalizado, pero que mantiene vínculos con la política tradicional. Por otra parte, podemos apreciar que los jóvenes toman distancia de esta forma de ejercicio de la política y el poder, resultando de ello una asimilación entre ellos mismos y aquellos actores no tradicionales y que buscan caminos no convencionales de acción (movimientos sociales).

Los jóvenes se identifican con la protesta, pero reclaman también para sí un sentido de comunidad. Se trata de una potencia opuesta, generada desde el mundo social, al poder (dominio) de los actores tradicionales, que se expresa por vías no institucionales y que utiliza formas de acción no convencionales. Se trata de una forma comunitaria de ejercicio de la política opuesta a la forma tradicional que ha tomado la democracia en nuestro entorno.

A continuación llevaremos las distancias obtenidas a un espacio de baja dimensionalidad (en nuestro caso de dos dimensiones) de modo de poder representar las proximidades en un mapa, lo que nos permitirá obtener información acerca de las dimensiones subyacentes de juicio utilizadas por la muestra para realizar la referida partición del campo por medio de los conceptos analizados. Lo anterior lo realizaremos por medio de un procedimiento de escalamiento multidimensional (índices de ajuste: Stress $=0.144$ y $\mathrm{RSQ}=0.889$ ). En la Figura 3 se muestran los resultados obtenidos. 
Figura 3

Escalamiento multidimensional

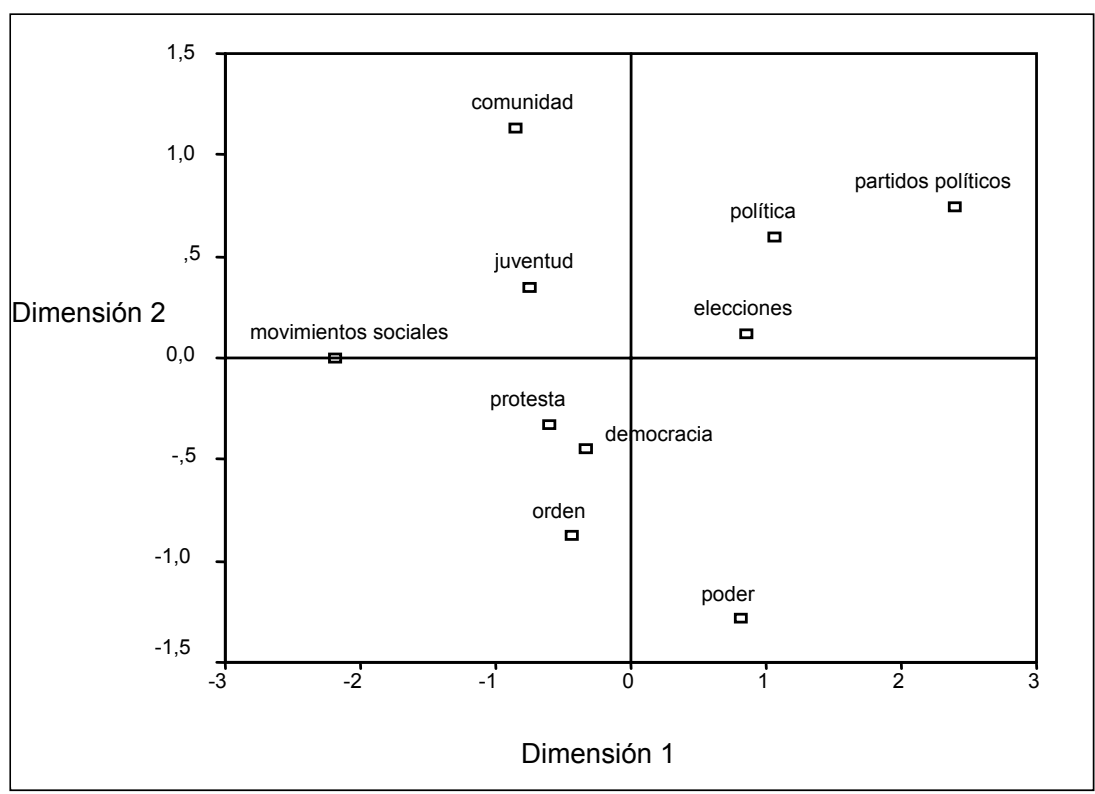

Como podemos apreciar, el eje uno opone los términos movimientos sociales y partidos políticos. Cercano al campo semántico de primer concepto se encuentran los términos comunidad y juventud. En el campo semántico de partidos políticos podemos encontrar los conceptos de política, elecciones y poder. Se trataría de un eje que aludiría a la dimensión de institucionalización y que nos indicaría una gradiente que va desde el máximo de formalismo en los procedimientos hasta una menor formalización y normatividad. Es decir, nuestra muestra tiende a utilizar una categoría que evalúa el grado de institucionalización de las prácticas y la cercanía respecto de los controles normativos, situándose a sí misma de un lado e indicándonos con ello su rechazo de los excesivos controles normalizadores de la acción colectiva y de la excesiva institucionalización de la actividad política. Por otra parte, y si atendemos a la segunda dimensión, vemos cómo se opone el concepto de comunidad a los de poder y orden. Es decir, lo que nos indica esta dimensión sería la idea de una comunidad que resiste $u$ opone su potencia al poder y el orden erigidos desde un lugar alejado de la misma. Se puede observar una crítica implícita a la idea de orden realmente existente, en tanto 
ajeno a la noción de comunidad y debido a que este emana de un poder que se expresa como dominio institucionalizado.

Ya sabemos cómo se posicionan los jóvenes en relación con los conceptos que se les han ofrecido para su evaluación. Nos corresponde ahora dar un paso más y generar análisis específicos sobre los estímulos «democracia» y «política». Para ello realizamos un análisis de correspondencias múltiples sobre los términos más frecuentemente asociados a ellos. Para el caso del estímulo «democracia» el número total de asociaciones ascendió a 110, las que se han reducido a 67 , de entre las cuales hemos escogido aquellas de más alta frecuencia (superior a cuatro) de aparición para utilizarlas como elementos activos para generar las dimensiones o factores subyacentes. Las tres primeras dimensiones aportan un $51.36 \%$ de la inercia total (o varianza) y son justamente estas las que utilizaremos para nuestra interpretación. En la Figura 4 podemos apreciar la gráfica para las dos primeras dimensiones.

Figura 4

Estímulo: democracia (dimensiones 1 y 2)

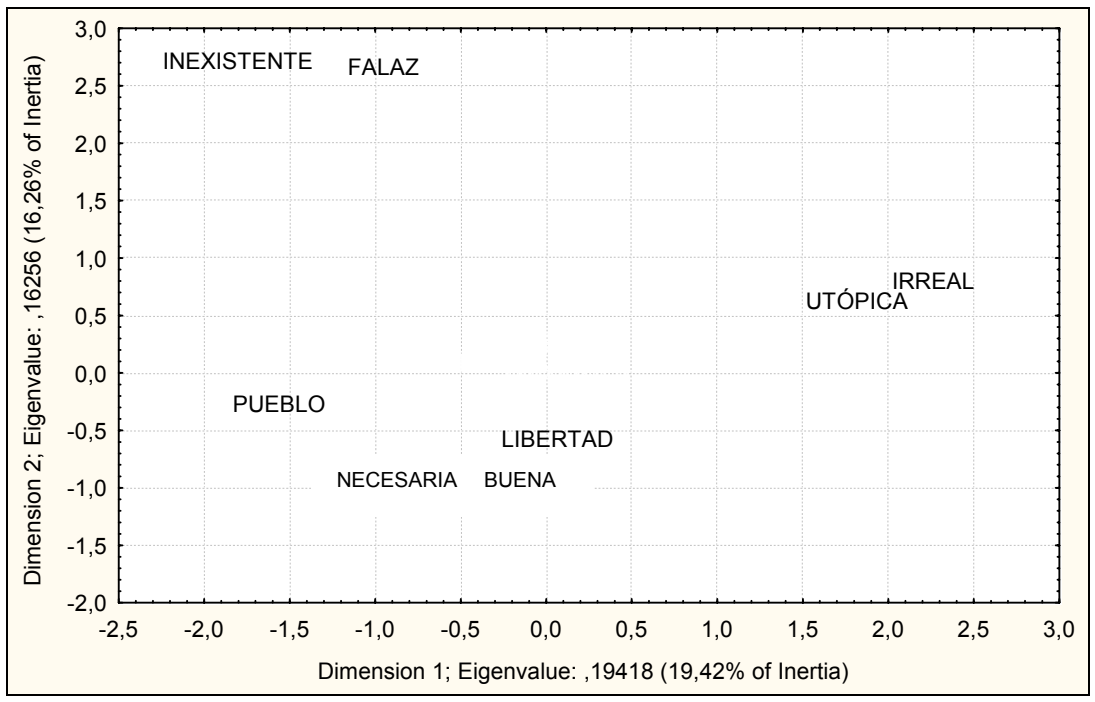

La primera dimensión (que aporta el $19.42 \%$ de la inercia) opone las palabras «inexistente» e «irreal», siendo parte del campo de representación de esta última la palabra «utópica». Esta dimensión nos podría estar indicando que los sujetos de la muestra evalúan la democracia como un 
ideal inexistente en la práctica real, toda vez que se pone en duda la verdadera posibilidad de que la democracia - tal cual como ellos la entienden - sea realmente posible de ser alcanzada en la práctica. Es decir, se pondría en cuestión no sólo la real existencia de ésta, sino además las reales posibilidades de construir una democracia ideal. Si atendemos al hecho de que junto a la palabra inexistente los sujetos sitúan la de pueblo, entonces podemos ampliar un poco nuestra interpretación y atribuir esta cercanía a la idea de que es justamente al pueblo a quien se le niega la democracia. También puede indicarnos que ante la inexistencia de la idea de «pueblo» la democracia no puede ser real y sólo puede existir en un sentido utópico (como idea regulativa). Si complementamos esta interpretación con los contenidos del factor dos $(16.26 \%$ de inercia) vemos a los jóvenes de nuestra muestra oponer las palabras «inexistente»y «falaz» con «necesaria»y «buena». De lo que se trata es justamente de que la democracia realmente existente se juzgaría como falsa y mentirosa, toda vez que se reconoce la necesidad de avanzar en sentido de la construcción de una democracia de diferente tipo y que sería evaluada como buena. Es decir, se trataría de una dimensión que articula principios de identidad y oposición (en tanto describe la democracia existente, toda vez que contiene implícitamente su crítica) con los de totalidad (el horizonte al que apunta su acción y que tiene que ver con la necesidad de construir una democracia «verdadera»).

Si atendemos ahora a la Figura 5, veremos que la dimensión tres (15.69\% de inercia) opone las palabras «libertad» y «pueblo». El campo semántico de la primera queda conformado por la palabra «falaz». Para «pueblo» se asocian los términos «necesaria»e «irreal». Esta dimensión viene a complementar los análisis anteriores en sentido de ratificar la idea de que tanto la idea de pueblo como la de las libertades asociadas a este serían engañosas. Los sujetos apuntan a cuestionar la idea de la existencia de un real pueblo, toda vez que apuntan a consagrar la necesidad de su formulación. Ante la inexistencia de una comunidad política, el discurso que apunta a resaltar su libertad sólo puede resultar en un engaño. La idea de libertad, tan bullada por tan diversos actores, no puede ser real sin la construcción de un referente comunitario dentro del cual tenga sentido. De este modo, se ponen bajo sospecha la idea de democracia en tanto esta sólo podría consistir en un ejercicio comunitario de las libertades, el cual no es posible al desdibujarse dicho actor comunitario. Eso sí, la democracia como idea regulativa, como horizonte, está en el centro de la reflexión de estos jóvenes.

Figura 5 
Estímulo: democracia (dimensiones 2 y 3)

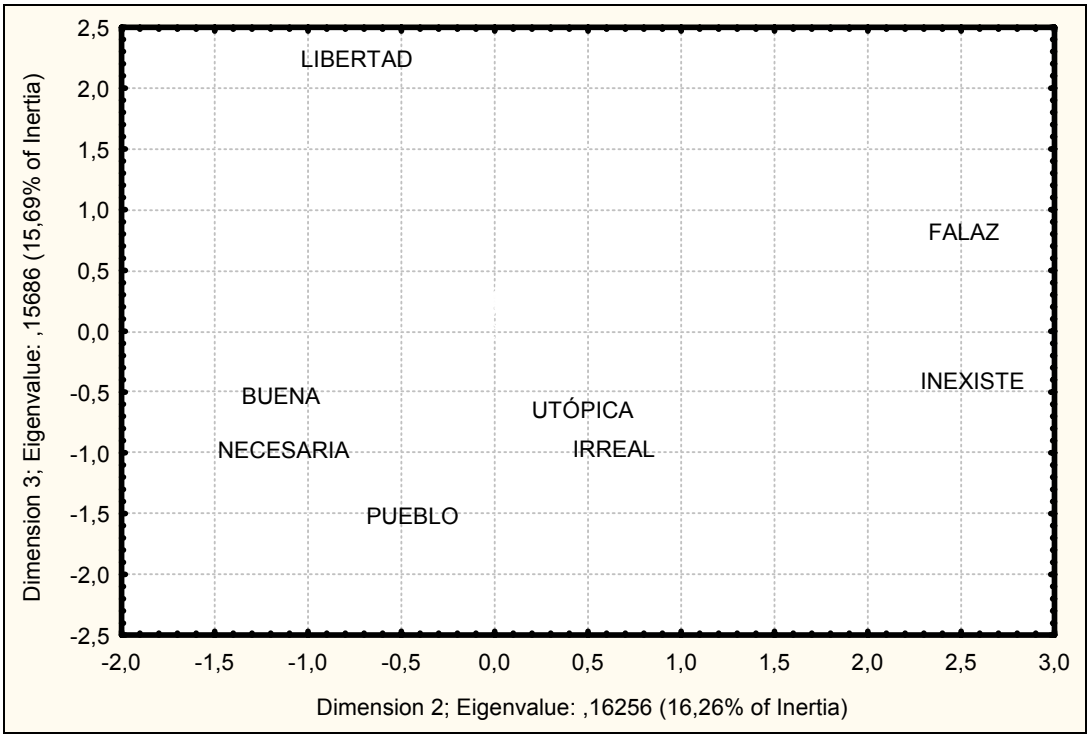

Figura 6

Estímulo: politica (dimensiones 1 y 2)

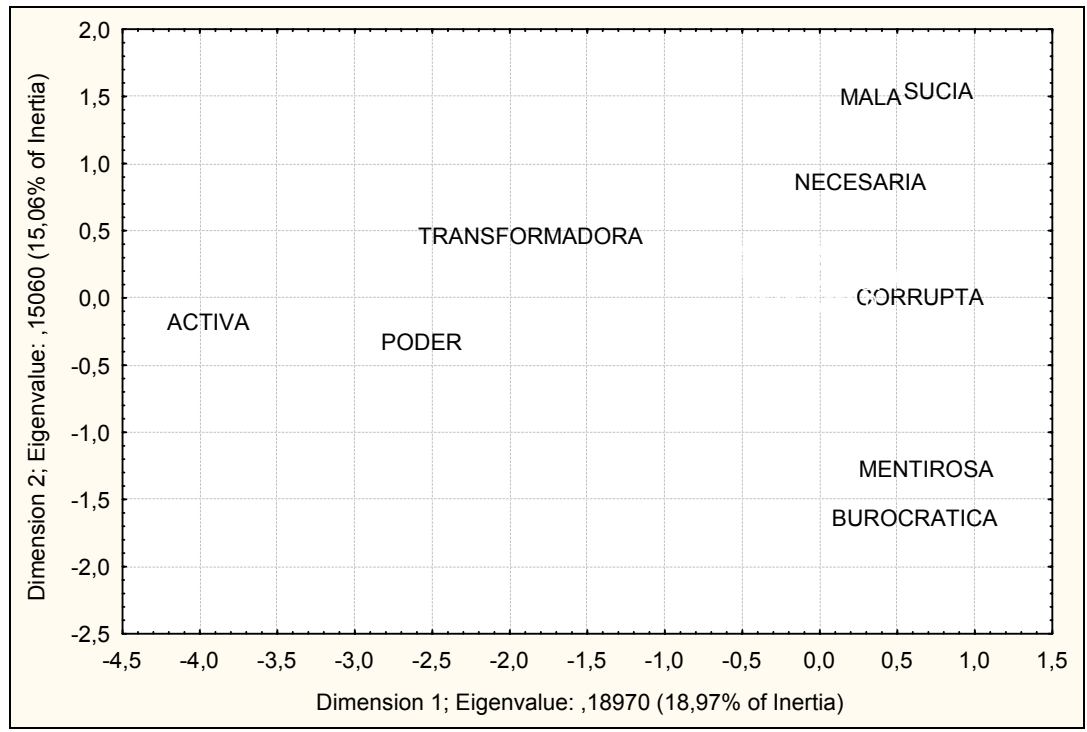

Avancemos un poco más y veamos cómo nuestra muestra se representa 
la política. Para ello hemos reducido el número total de asociaciones de 149 conceptos a 75, y hemos seleccionado aquellos con frecuencia de aparición más elevada. En conjunto, las tres dimensiones seleccionadas (Figuras 6 y 7) explican un $49.87 \%$ de la inercial total.

Como podemos apreciar, la primera dimensión para el término «política» opone las palabras «activa» con una serie de palabras que evalúan dicho concepto (corrupta, mentirosa, burocrática, sucia y mala). Claramente esta dimensión apunta a dar cuenta de la impresión general que los participantes tienen de la política, y donde ésta no sale bien parada. Pero también nos entrega del otro lado la idea de la necesidad de oponerse activamente a ella (aunque relativamente lejanas de «activa» se encuentran las palabras «poder» y «transformadora») por medio de una acción transformadora que dé cuenta del poder de los actores sociales mantenidos al margen de la política profesional.

Figura 7

Estímulo: política (dimensiones 2 y 3 )

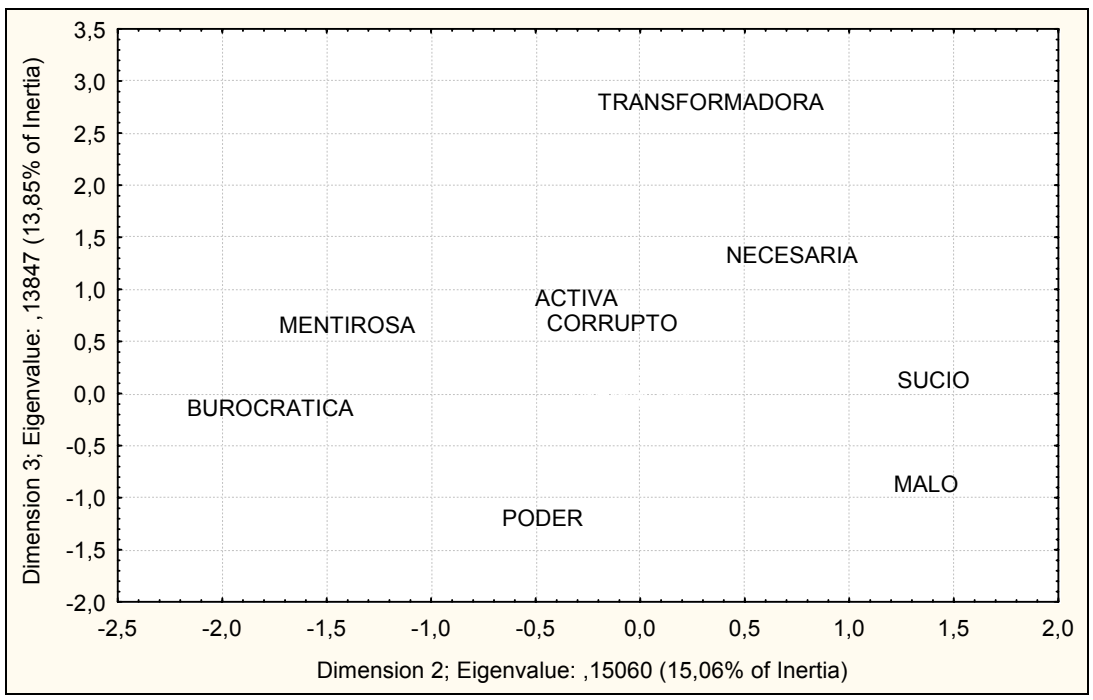

El factor dos no nos entrega nada nuevo, salvo que se resalta la negativa evaluación que se hace de la actividad política (se oponen las palabras «mala» y «sucia» con «burocrática» y «mentirosa»). Ahora bien, no debe pasarnos desapercibida la aparición en ambos factores y dentro de los polos que evalúan negativamente la política de la palabra 
«necesaria», indicándonos que aún en los contextos más nocivos la política es una actividad ineludible. Es decir, aún totalmente desvirtuada y encontrándose absolutamente desvalorada la política se resalta la necesidad de habérselas con ella - aunque quizás ya no como actividad sino como plano de existencia-. En este sentido, es extremadamente relevante destacar que nuestros jóvenes lejos de alejarse de la política se restan o marginan de una forma de entenderla: aquella que la asimila con la política profesional de corte burocrático.

Si nos centramos ahora en la tercera dimensión o factor, podremos apreciar que el eje opone los conceptos «transformadora» (cuyo campo de representación queda conformado por «necesaria» y «activa») con «poder» (que queda acompañado por «burocrática» $\mathrm{y}$ «malo»). Es decir, este eje viene a reforzar la idea de que frente al poder burocrático negativamente evaluado se debe oponer una necesaria acción transformadora. Lo anterior nos indica que los jóvenes no dejan a su suerte la actividad política y sus vicios, sino que de algún modo intentan oponer la idea de que lo político es un campo donde al dominio institucionalizado (campo donde circula y se ejerce el poder) se puede oponer una potencia, comunitariamente articulada que contribuya decididamente a la construcción de una democracia en la que creen, pero que nada tiene que ver con aquella que se les ofrece y tiene existencia práctica.

Esta interpretación es totalmente coherente con los datos que ya hemos aportado sobre la escasa o nula confianza que los jóvenes manifiestan hacia las instituciones y sus tradicionales canales de participación. El diferencial semántico también apoya estas interpretaciones en sentido de ratificar la idea de los jóvenes de la necesidad e importancia de la actividad política, toda vez que nos muestran su desencanto y distancia respecto de la actividad que se les ofrece y que juzgan como ineficaz, carente de dinamismo, rígida e intolerante, centralizada y lejana, entre otras. Lo que se critica es la forma que ha tomado la actividad, su carácter excluyente y negador de las diferencias existentes en el seno de lo social, su nula capacidad para incluir a la mayoría en las ventajas del sistema y de respetar la riqueza que la diversidad de opciones y estilos de vida significa, de lo que tiene de castradora para una generación que maneja códigos diferentes de relación con lo institucional y que concibe lo político como un ámbito comunitario en donde todos tienen derecho a decidir sobre aquellas cosas que les afectan, donde todos tienen control sobre la gestión de los asuntos relevantes para la comunidad de la que participan y derecho a partici- 
par activamente en la construcción de sus vidas. Todo ello en el marco de una democracia participativa (y por tanto con elementos que apuntan al agotamiento de la representación como modo privilegiado de ejercicio de la actividad política).

\section{DISCUSIÓN}

Una representación social es una forma de pensamiento construida comunitariamente mediante procesos de comunicación grupal. Se trata del denominado «pensamiento del sentido común». Ahora bien, decíamos que tiene un carácter práctico en tanto prescribe comportamientos a los miembros del grupo y en tanto permite que en esas prácticas sus integrantes puedan encontrar un soporte de su identidad. Una representación integra la información que circula en el medio social y permite hacer inteligible el entorno en que tiene lugar la convivencia entre grupos. Para el caso que analizamos, podemos considerar que se trata de una interpretación realizada sobre el contexto en el que tiene lugar la interacción y que permite realizar un mapa del territorio en que a los jóvenes les toca moverse.

Lejos de las interpretaciones que nos presentan a la juventud como retraída sobre sus propios intereses individuales o a la burda imagen de una juventud entregada al hedonismo y la apatía, nos encontramos con actores sociales que poseen una visión clara de lo que está en juego cuando lo que se hace saliente es la idea de política. Se trata de sujetos que más que retraerse han tomado distancia de una forma de entender la política y que han optado por construir en su espacio comunitario y en sus relaciones interpersonales aquello que les es negado desde arriba. Jóvenes que han emprendido una travesía que les permita acumular legitimidad, que les permita ejercitar cotidianamente los contenidos que sienten vinculados a la actividad política en una democracia construida a otra escala (una democracia de pares y donde el otro no es un sujeto abstracto sino con nombre propio). De allí que podamos afirmar que actividades no consideradas como tradicionalmente políticas (grupos culturales, colectivos artísticos, grupos de amistad o esquina, clubes deportivos y en general todos los espacios comunitarios de pequeña escala) hoy son un laboratorio práctico de los contenidos que trae aparejada una nueva forma (aún incipiente quizás) de entender la democracia y la política.

Lo anterior no habla, además, del nuevo lugar desde donde se nos 
propone implementar las transformaciones que juzgan necesarias. Se trata de un espacio más informal, que contiene menos controles normativos y donde las jerarquías son reducidas a su mínima expresión. Se trata de vivir cotidianamente esas transformaciones y de acumular una potencia que se oponga desde abajo al poder institucionalizado. Un poder que es vivido como dominio desde arriba, cuya legitimidad es cuestionada y del que ya no se espera el cumplimiento de las transformaciones ofrecidas. Parece haber un aprendizaje y tiene que ver con que una vez que se ha ingresado al poder institucional, cuando se han aprendido sus códigos y se ha cedido ante sus exigencias, entonces el actor político es quien resulta transformado. De allí la excesiva distancia que intentan mantener del espacio institucional.

Para el discurso que venimos analizando la idea de cambio es rectora de su pensamiento, la idea de democracia sigue siendo una idea regulativa fundamental, la actividad política sigue poseyendo actualidad y relevancia. Lo que parece haber cambiado son los contenidos asociados a dichos conceptos: una democracia más participativa, una idea de la actividad política vinculada a la autogestión comunitaria de la vida, una idea de que las transformaciones se juegan en el espacio local donde tiene lugar la vida cotidiana.

Estamos en presencia de un grupo de jóvenes que ha generado un universo consensual de sentido, que ha construido un espacio en los pliegues del sistema desde donde vivir cotidianamente con autonomía. Se trata de un proyecto comunitario y desde los márgenes de la políti$\mathrm{ca}$, de un proyecto que aunque no explicitado contiene un potencial emancipador. Ciertamente se trata de una minoría, pero bien sabemos que todas las grandes transformaciones han comenzado simplemente por ser prácticamente contranormativas, por constituirse como el fermento social sobre cuyo estilo de relación la sociedad en su conjunto puede centrar la mirada. Se trata de un proyecto práctico, comunitariamente articulado, la mayor parte de las veces implícito, pero que puede tener como efecto no intencionado la construcción de una potencia desde abajo que se oponga a un sistema desgastado y con severos cuestionamientos a su legitimidad. La novedad siempre en la historia ha venido aparejada a las minorías.

Finalmente, sólo nos queda señalar que contra nuestras expectativas los jóvenes han generado representaciones sumamente similares entre sí, independientemente de su sexo, afiliación política, ideología o nivel socioeconómico. Es decir, la categoría «jóvenes» parece funcionar para el caso de los objetos evaluados con independencia de 
criterios tradicionalmente relevantes. Lo que nos queda por comprobar aún es si esta aparente identidad de la representación lo es realmente, para lo cual en futuros estudios deberíamos centrarnos en la comparación de los núcleos de las representaciones elaboradas, cuestión que con los procedimientos que hemos escogido en este estudio no podemos determinar.

ANTOFAgASTA (CHILE), ABRIL 2007

RECIBIDO: ABRIL 2007

ACEPTADO: JUNIO 2007

\section{REFERENCIAS BIBLIOGRÁFICAS}

CÁRDENAS, M. (2006): El análisis multivariante de las representaciones sociales. Antofagasta: Editorial Universidad Católica del Norte.

— y A. BlANCO (2006): «Representación e influencia de los nuevos movimientos sociales». Revista de Psicología social.

— $\mathrm{y}$ - (2004): «Las representaciones sociales del movimiento antiglobalización». Psicología política.

CARUgati, F. y A. Palmonari (1991): A propósito de las representaciones sociales. Anthropos.

DÁviLA, O. (2004): «Adolescencia y juventud: de las nociones a los abordajes». Última Década $\mathrm{N}^{\circ} 21$. Valparaíso: Ediciones CIDPA.

Di Giacomo, J-P. (1987): «Teoría y método de análisis de las representaciones sociales». En D. PÁEZ (editor): Pensamiento, individuo y sociedad. Cognición y representación social. Madrid: Fundamentos.

_ (1980): «Intergroup alliances and rejections within a protest movement (Analysis of social representations)». European Journal of Social Psychology.

DOISE, W. (1991): Las representaciones sociales: presentación de un campo de investigación. Anthropos, suplementos.

—, A. CLÉMENCE y F. LORENCI-CIOLDI (1993): The quantitative analysis of social representations. Londres: Harvester Wheatsheaf.

ECHEBARRÍA, A. y J. ÁlVAREZ (1996): «Representaciones sociales de la democracia y el sistema electoral: estudio comparativo entre México y el País Vasco». Revista de Psicología Social, 11 (1).

— M. MERNÁNDEZ y L. GONZÁLEZ (1993): «Social representations and intergroup conflicts: who's smoking here». European Journal of Social Psychology.

GALAM, S. y S. MoscovicI (1991): «Toward a theory of collective phenom- 
ena. Consensus and attitude changes in group». European Journal of Social Psychology.

Garretón, M. A. y T. Villanueva (1999): «Política y jóvenes en Chile». Santiago: Corporación Participa y Fundación Ebert.

INSTITUTO NACIONAL DE LA JUVENTUD (2003): IV encuesta nacional de juventud. Santiago: INJUV.

JODELET, D. (1986): «La representación social: fenómenos, concepto y teoría». En S. Moscovici (editor): Introducción a la psicología social. Barcelona: Paidós.

MoscovicI, S. (1984): «The phenomenon of social representations». En R. FARR y S. MOSCOVICI (editores): Social Representations. Londres: Cambridge University Press.

_ (1979): El psicoanálisis, su imagen y su público. Buenos Aires: Huemul.

OsGoOd, Ch.; G. SUCI y P. TANEMBAUM (1976): La medida del significado. Madrid: Editorial Gredos.

SANDOVAL, J. (2003): «Ciudadanía y juventud: el dilema entre la integración social y la diversidad cultural». Última Década $\mathrm{N}^{\circ} 19$. Valparaíso: Ediciones CIDPA. 